\title{
Dieta de la rana de hojarasca Craugastor rhodopis (Anura: Craugastoridae): una especie abundante en la región montañosa del este de México
}

\author{
Ricardo Luría-Manzano, Marco Tulio Oropeza-Sánchez, José Luis Aguilar-López*, \\ Juan Manuel Díaz-García \& Eduardo Pineda \\ Red de Biología y Conservación de Vertebrados, Instituto de Ecología, A. C., Carretera antigua a Coatepec No. 351, \\ El Haya, CP. 91070, Xalapa, Veracruz, México; mtos0290@gmail.com, jlal.herp@gmail.com, \\ juanm.diazgarcia@gmail.com, eduardo.pineda@inecol.mx \\ * Correspondencia
}

Recibido 07-V-2018. Corregido 30-VIII-2018. Aceptado 21-I-2019.

\begin{abstract}
Diet of the leaf litter frog Craugastor rhodopis (Anura: Craugastoridae): an abundant species in the mountainous region of the east Mexico. Dietary composition in amphibians may be influenced by several factors that cause their intraspecific variation, such as geographic distribution, ontogeny, seasonality, and sex of organisms. The objective of this study was to analyze the composition of the diet in adults of the Polymorphic Robber Frog Craugastor rhodopis in the mountainous region of central Veracruz, Mexico. A total of 77 adult individuals were collected during the rainy season of 2012, of which 66 had stomach contents. We performed an analysis of the intersexual variation in diet diversity, volume, number of prey, and number of prey categories consumed. The diet of $C$. rhodopis was composed by 20 categories. Orthoptera predominated in terms of number, volume, frequency of occurrence, and relative importance. Males consumed smaller prey items relative to females. Although both sexes consumed similar numbers of prey, we found a significant effect of interaction of sex and size of frogs on this variable, with small females consuming more prey than the large ones, whereas in males the opposite occurred. High consumption of orthopterans (at least in volume) is a common phenomenon within Craugastoridae and is possibly related to the success of some species in the ecosystems where they live. Although the composition of the diet was similar between sexes in C. rhodopis, we suggest that intersexual competition for trophic resources is minimal, given differences in prey size consumed by males and females.
\end{abstract}

Key words: amphibians; trophic ecology; dietary composition; trophic diversity; linear models; intersexual variation.

Luría-Manzano, R., Oropeza-Sánchez, M. T., Aguilar-López, J. L., Díaz-García, J. M. \& Pineda, E. (2019). Dieta de la rana de hojarasca Craugastor rhodopis (Anura: Craugastoridae): una especie abundante en la región montañosa del este de México. Revista de Biología Tropical, 67(1), 196-205.

Estudiar la dieta de los anuros ayuda a reconocer parte de las funciones de este grupo biológico en los ecosistemas (Norval, Huang, Mao, Goldberg, \& Yang, 2014), principalmente disminuyendo la abundancia de algunos grupos de invertebrados y la herbivoría foliar (Beard, Eschtruth, Vogt, Vogt, \& Scatena, 2003). Además, amplía el conocimiento sobre sus historias de vida y la manera en que los organismos utilizan los recursos para asignar energía al crecimiento, al mantenimiento y a la reproducción (Zug, Vitt, \& Caldwell, 2001). El estudio de los hábitos alimenticios de los anuros puede resultar complejo, ya que los sitios (Bonansea \& Vaira, 2007) y la estacionalidad (Berazategui, Camargo, \& Maneyro, 2007) pueden ofrecer 
recursos tróficos distintos. Asimismo, debido a la ontogenia (Whitfield \& Donnelly, 2006) y el sexo (Maneyro, Naya, da Rosa, Canavero, \& Camargo, 2004), los anuros pueden presentar diferentes requerimientos alimenticios. Estos factores pueden provocar variaciones intraespecíficas en la composición de la dieta, e influir en la estrategia de forrajeo (Lima \& Magnusson, 2000).

La variación intersexual en la dieta de las especies se ha relacionado con la evolución del dimorfismo sexual, específicamente con el tamaño de la cabeza (Houston \& Shine, 1993), el período y el comportamiento reproductivo (Lamb, 1984; Donnelly, 1991), los requerimientos energéticos para la producción de gametos (Bull, 2003) y la divergencia en la selección de hábitat (Plummer \& Farrar, 1981). La variación intersexual en la dieta de anuros es conocida para pocas especies, entre las cuales se encuentran ránidos (Lamb, 1984), leptodactílidos (Maneyro et al., 2004), hílidos (Miranda, Ebner, Solé, \& Kwet, 2006) y bufónidos (Quiroga, Sanabria, \& Acosta, 2009); no existiendo casos documentados para la familia Craugastoridae, pese a que es la familia con el mayor número de especies a nivel mundial (Frost, 2018).

Dentro de la familia Craugastoridae, el género Craugastor está compuesto por 115 especies y se distribuye desde el sur de Estados Unidos hasta el noroeste de Colombia y Ecuador, a través de hábitats tropicales y subtropicales (Frost, 2018). Todos los miembros de este género se caracterizan por tener desarrollo directo y varias especies se encuentran entre las más abundantes en los ecosistemas donde habitan (Lieberman, 1986; Urbina-Cardona, Olivares-Pérez, \& Reynoso, 2006; RodríguezMendoza \& Pineda, 2010), por lo que podrían tener un papel importante en la red trófica y la estructura de las comunidades (Urbina-Cardona \& Reynoso, 2009). Los estudios de dieta en especies de la familia Craugastoridae se han centrado en el género Pristimantis (Arroyo, Serrano-Cardozo, \& Ramírez-Pinilla, 2008; Garcia, Posso-Gómez, \& Cárdenas-Henao, 2015), mientras que otros como Craugastor han recibido menor atención, a pesar de su amplia distribución geográfica y el elevado número de especies que lo componen.

Craugastor rhodopis (Cope, 1867) es una especie que alcanza los $42.3 \mathrm{~mm}$ de longitud hocico-cloaca (LHC) y presenta dimorfismo sexual en la talla, siendo las hembras ( $\mathrm{LHC}=$ 36.7 - $42.3 \mathrm{~mm}$ ) más grandes que los machos $(\mathrm{LHC}=23.8-30.4 \mathrm{~mm})$ (Streicher et al., 2014). Esta especie es endémica a México y se distribuye aproximadamente entre los 1260 y $1860 \mathrm{msnm}$, en bosques de encino-pino y bosques de niebla en los estados de San Luis Potosí, Hidalgo, Puebla y Veracruz (Streicher et al., 2014), en la Sierra Madre Oriental de México. Craugastor rhodopis tiene hábitos terrestres y está asociada a sitios con abundante cobertura arbórea y hojarasca. A pesar de ser una especie con alta abundancia en los ensambles de anfibios de los remanentes de bosque donde habita (Murrieta-Galindo, López-Barrera, GónzalezRomero, \& Parra-Olea, 2013; Meza-Parral \& Pineda, 2015), existe poca información acerca de diferentes aspectos de su historia natural e interacción con otras especies (Aguilar-López \& Pineda, 2013). Dado que C. rhodopis presenta dimorfismo sexual, es posible que exista variación intersexual en su dieta.

En este estudio se evaluó la composición de la dieta consumida por adultos de C. rhodopis, analizando específicamente si existen diferencias entre sexos en el volumen, la frecuencia y la abundancia de las presas consumidas, y la combinación de estos tres atributos (índice de importancia relativa). Además, se evaluó la relación de la talla y el sexo de las ranas con el volumen, la cantidad de presas y el número de categorías de presa consumidas.

\section{MATERIAL Y MÉTODOS}

Área y sitios de estudio: El área de estudio se localiza en la región montañosa central de Veracruz (19³0'12'- 1902'22" N $\left.\& 96^{\circ} 48^{\prime} 15^{\prime \prime}-97^{\circ} 08^{\prime} 10^{\prime \prime} \mathrm{W}\right)$ en un intervalo altitudinal entre 1000-1950 msnm, en la Sierra Madre Oriental. La topografía del área de estudio es heterogénea, el clima templado-húmedo 
con una temperatura media anual entre 12 y $18{ }^{\circ} \mathrm{C}$ y una precipitación total anual que varía entre 1350 y $2000 \mathrm{~mm}$ (Williams-Linera, 2007). El área, originalmente cubierta por bosque de niebla, actualmente está conformada por algunos fragmentos de bosque inmersos en una matriz de ambientes modificados (WilliamsLinera, Manson, \& Isunza, 2002). Para llevar a cabo las búsquedas de las ranas, se seleccionaron ocho fragmentos de bosque de niebla, con un área entre 0.09 y $3.53 \mathrm{~km}^{2}$, en los cuales la presencia de la especie ya ha sido confirmada (Meza-Parral \& Pineda, 2015).

Recolecta de los ejemplares y análisis del contenido estomacal: Para la captura de los individuos de $C$. rhodopis se realizaron muestreos nocturnos (20:00 - 03:00 h) entre junio y octubre de 2012 (época de lluvias), utilizando la técnica de búsqueda libre con encuentros visuales (Crump \& Scott, 1994). Todos los individuos fueron sacrificados con pentobarbital sódico, en un periodo máximo de 4 horas después de su captura (Garcia et al., 2015). Los ejemplares fueron depositados en la Colección de Anfibios y Reptiles del Instituto de Ecología A. C. (CARIE). De cada ejemplar se midió la longitud hocico-cloaca (LHC) y se extrajeron las gónadas para determinar su sexo, y el estómago. No se consideraron las presas del intestino, para permitir comparar con la mayoría de los estudios de dieta en craugastóridos, que solo analizan las presas del estómago. Este estudio se limitó a individuos adultos, a los cuales se les determinó el sexo con base en la presencia de testículos bien desarrollados en machos, y folículos u oviductos convolutos en hembras. Los ejemplares fueron recolectados con los permisos otorgados por la Dirección General de Vida Silvestre de la Secretaría de Medio Ambiente y Recursos Naturales (SGPA/ DGVS/03665/06 y SGPA/DGVS/03444/15).

El contenido estomacal fue analizado empleando un microscopio estereoscópico, las presas encontradas fueron identificadas a nivel de orden con ayuda de las claves de Triplehorn \& Johnson (2005). En insectos holometábolos, adultos y larvas fueron considerados categorías de presa distintas, y los himenópteros fueron separados en formícidos y no formícidos (Whitfield \& Donnelly, 2006). Se contó el número de presas presentes en cada estómago, y se midió su largo y ancho con un calibrador digital $(0.01 \mathrm{~mm})$, para posteriormente calcular el volumen de cada una de estas con la fórmula del elipsoide (Crawford, Shepard, \& Conner, 2009).

Análisis de datos: Para determinar la importancia de cada categoría de presa $i$ en la dieta de la especie (en conjunto y por sexo), se tomaron en cuenta tres variables: 1) el porcentaje volumétrico de las presas $(\% \mathrm{~V}) ; 2)$ el porcentaje de frecuencia de ocurrencia ( $\% \mathrm{FO})$; 3) el porcentaje numérico de las presas consumidas $(\% \mathrm{~N})$. Posteriormente, se calculó el índice de importancia relativa (IIR) propuesto por Biavati, Wiederhecker y Colli (2004). Para comparar cada variable y el IIR de las categorías de presas entre hembras y machos, se utilizaron gráficas de rango-abundancia (Magurran, 2004). Con el fin de comparar el número de categorías de presas entre machos y hembras se generaron curvas de rarefacción para cada sexo, basándonos en el número de individuos de cada categoría de presa, empleando el programa EstimateS 9.0 (Colwell, 2013). Debido a la diferencia en el número de individuos entre sexos, se realizó una extrapolación de la curva de las hembras (48 individuos), al nivel de la muestra de machos (133 individuos) con mayor número de individuos (Colwell et al., 2012) y posteriormente se recalcularon los intervalos de confianza al $84 \%$ que mimetizan una prueba estadística con un valor de alfa de 0.05 (MacGregor-Fors \& Payton, 2013). Se analizó la sobreposición de la dieta entre sexos con el índice de Pianka (1973), considerando los datos de abundancia y el volumen de las presas por separado. Adicionalmente, se evaluó la variación en la composición de la dieta entre sexos con un análisis de similitud (ANOSIM), basado en una matriz de disimilitud de BrayCurtis (Clarke, 1993).

Para determinar si existe relación entre el tamaño (LHC), el sexo de los ejemplares, 
o una interacción entres estos factores con el volumen, el número de presas y el número de categorías de las presas fue empleado un modelo lineal mixto, en el caso del volumen, y modelos lineales generalizados para el número de presas y número de categorías de presas. Para el modelo lineal mixto se empleó una función de liga raíz cuadrada, considerando los efectos fijos de la identidad de cada individuo (Crawley, 2007). Para el número de categorías de presas y el número de presas, los modelos lineales generalizados (GLM) fueron generados empleando una distribución Gamma, con la función de liga raíz cuadrada. Todos los análisis fueron realizados en $\mathrm{R}$ versión 3.1.3 ( $\mathrm{R}$ Development Core Team, 2015) y sus extensiones gmodels (Warnes, 2006) y lme4 (Bates, Maechler, Bolker, \& Walker, 2015).
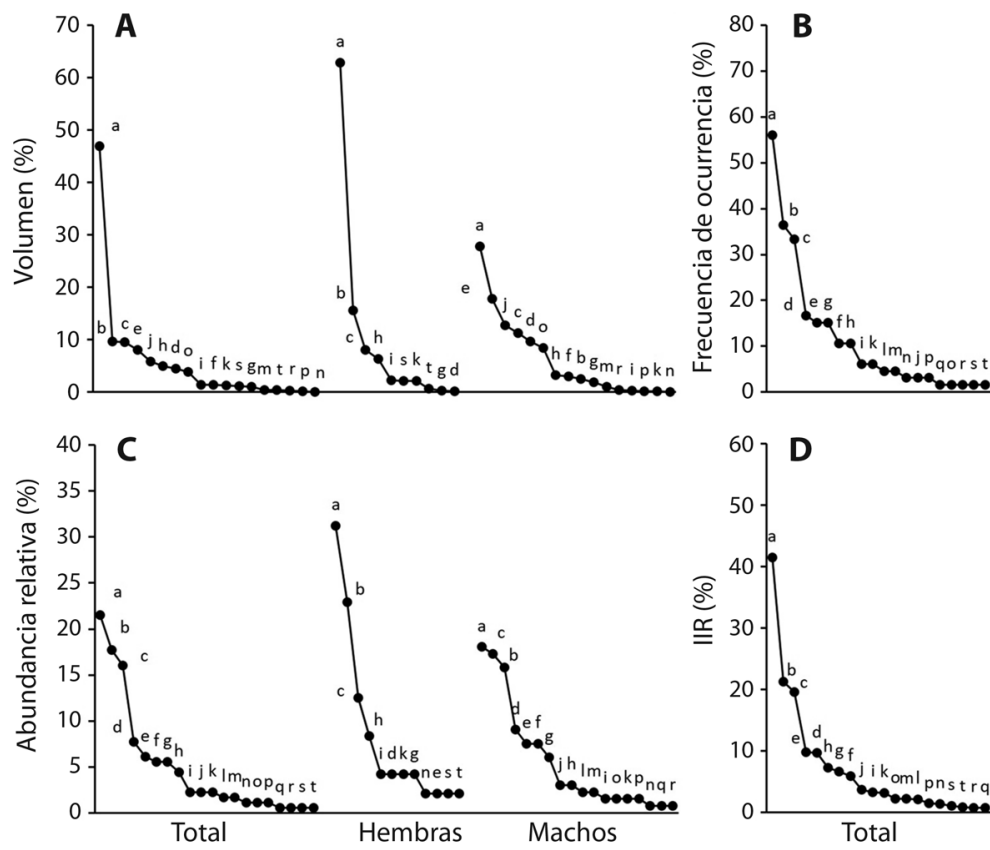

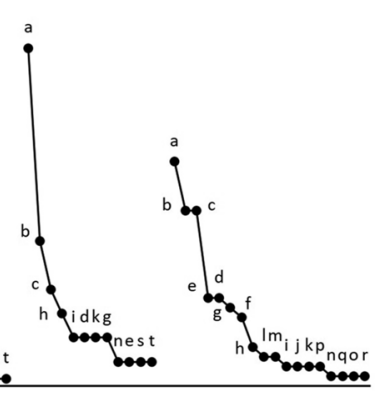

\section{RESULTADOS}

En total se recolectaron 77 ejemplares adultos (56 machos y 21 hembras) de C. rhodopis, con talla entre 26.3 y $44.3 \mathrm{~mm}$, siendo las hembras en promedio $30 \%$ más grandes $(\overline{\mathrm{X}} \pm$ ee $=37.2 \pm 3.7 \mathrm{~mm}$, intervalo $=26-45.89 \mathrm{~mm}$ ) que los machos ( $\overline{\mathrm{X}} \pm \mathrm{ee}=28.7 \pm 4.8 \mathrm{~mm}$, intervalo $=20.15-37.51 \mathrm{~mm})$. Se encontró contenido estomacal en 66 individuos (47 machos y 19 hembras). En total se registraron 181 presas pertenecientes a 20 categorías (18 en machos y 12 en hembras). Fue posible determinar el volumen sólo de 101 presas contenidas en estómagos de 52 individuos (16 hembras y 36 machos). Orthoptera fue la categoría predominante en cuanto a volumen, frecuencia de ocurrencia, número e importancia relativa,

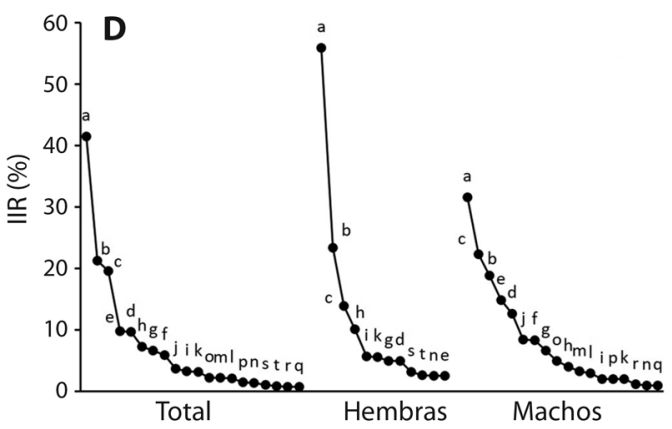

Fig. 1. Rango-abundancia con el porcentaje de volumen (A), de frecuencia de ocurrencia (B), de abundancia (C), e índice de importancia relativa (D), del total de la muestra ( $\mathrm{T}$ ) y de cada sexo $(\mathrm{H}=$ hembras, $\mathrm{M}=$ machos). $\mathrm{a}=$ Orthoptera, $\mathrm{b}=$ Araneae, $\mathrm{c}=$ Coleoptera, $\mathrm{d}=$ Diplopoda, $\mathrm{e}=$ Chilopoda, $\mathrm{f}=$ Dermaptera, $\mathrm{g}=$ Hymenoptera $($ Formicidae) $\mathrm{h}=\mathrm{Isopoda}, \mathrm{i}=$ Coleoptera (larva), $\mathrm{j}=$ Hemiptera, $\mathrm{k}=$ Hymenoptera (no Formicidae), $\mathrm{l}=$ Diptera, $\mathrm{m}=$ Lepidoptera $($ larva), $\mathrm{n}=\mathrm{Acari}, \mathrm{o}=$ Diptera (larva), $\mathrm{p}=$ Opilion, $\mathrm{q}=$ Decapoda, $\mathrm{r}=$ Homoptera, $\mathrm{s}=$ Neuroptera y $\mathrm{t}=$ Neuroptera (larva).

Fig. 1. Rank-abundance with the percentage of volume (A), frequency of occurrence (B), abundance (C), and relative importance index (D), of total of the sample $(\mathrm{T})$ and each $\operatorname{sex}(\mathrm{H}=$ females, $\mathrm{M}=$ males). $\mathrm{a}=$ Orthoptera, $\mathrm{b}=\mathrm{Araneae}, \mathrm{c}=$ Coleoptera, $\mathrm{d}=$ Diplopoda, $\mathrm{e}=$ Chilopoda, $\mathrm{f}=$ Dermaptera, $\mathrm{g}=$ Hymenoptera (Formicidae), $\mathrm{h}=$ Isopoda, $\mathrm{i}=$ Coleoptera (larva), $\mathrm{j}=$ Hemiptera, $\mathrm{k}=$ Hymenoptera (non Formicidae), $\mathrm{l}=$ Diptera, $\mathrm{m}=$ Lepidoptera (larva), $\mathrm{n}=$ Acari, $\mathrm{o}=$ Diptera (larva), $\mathrm{p}=$ Opilion, $\mathrm{q}=$ Decapoda, $\mathrm{r}=$ Homoptera, $\mathrm{s}=$ Neuroptera $\mathrm{y} \mathrm{t}=$ Neuroptera (larva). 
seguida por Coleoptera y Araneae. Este patrón se observó al analizar toda la muestra en conjunto, así como al separarlas por sexo (Fig. 1). El número de categorías de presas no difirió entre sexos, ya que se observa sobrelapamiento entre los intervalos de confianza al $84 \%$ de las curvas de acumulación (Fig. 2). En los machos se encontraron ocho categorías de presa que no se encontraron en las hembras: Hemiptera, Dermaptera, Diptera (adultos y larvas), Lepidoptera (larvas), Opilionida, Homoptera y Decapoda. Sin embargo, ninguna de estas estuvo entre las cinco presas más importantes en machos, por lo que se sugiere que su presencia se debe al mayor tamaño de muestra en estos. Las presas del orden Neuroptera (adultos

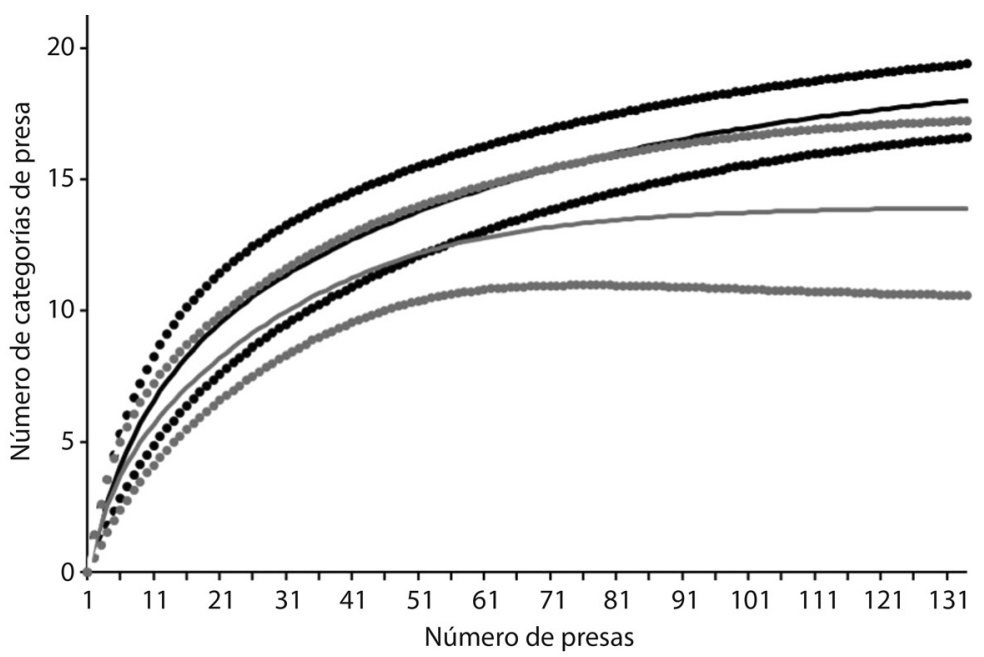

Fig. 2. Curvas de acumulación que muestran el número de categorías de presa esperado e intervalos de confianza al $84 \%$ para machos (líneas continua y punteadas de color negro) y hembras (líneas continua y punteadas de color gris).

Fig. 2. Accumulation curve showing expected number of prey categories and $84 \%$ confidence intervals for males (continuous and dotted black lines) and females (continuous and dotted gray lines).

y larvas) fueron encontradas únicamente en los estómagos de las hembras. Sin embargo, no se encontraron diferencias significativas en la composición de la dieta entre sexos, considerando los datos de abundancia $(\mathrm{R}=-0.01$; $\mathrm{P}=0.52)$ y volumen $(\mathrm{R}=0.02 ; \mathrm{P}=0.16)$.

El volumen promedio de las presas fue de $31.6 \mathrm{~mm}^{3}$ ( ee $=62.7 \mathrm{~mm}^{3}$, intervalo $=0.1-365$ $\mathrm{mm}^{3}$ ), el volumen de las presas encontradas en hembras osciló entre 0.1 y $365 \mathrm{~mm}^{3}(\overline{\mathrm{X}} \pm$ ee $\left.=53.54 \pm 90.2 \mathrm{~mm}^{3}\right)$ y en machos éste varió entre 0.1 y $226 \mathrm{~mm}^{3}$ ( $\overline{\mathrm{X}} \pm$ ee $\left.=22 \pm 43 \mathrm{~mm}^{3}\right)$. Se encontró una relación positiva entre la talla (LHC) de los individuos y el volumen de las presas $\left(\right.$ coef \pm ee $=0.32 \pm 0.06 ; \chi^{2}=18.2$, g.l. $=4 ; \mathrm{P}<0.001)$ y se observaron diferencias entre sexos. En los estómagos de las hembras las presas encontradas fueron de mayor volumen $\left(\chi^{2}=9.5\right.$, g.l. $\left.=4 ; \mathrm{P}=0.002\right)$. Pero no se observó un efecto de la interacción entre la talla y el sexo de las ranas $\left(\chi^{2}=4.67 ; \mathrm{gl}=6 ; \mathrm{P}=0.1\right.$; Fig. 3A).

El número de presas consumidas por los ejemplares de $C$. rhodopis varió entre uno y nueve $(\overline{\mathrm{X}} \pm$ ee $=2.8 \pm 1.7)$, en hembras osciló entre uno y siete $(\overline{\mathrm{X}} \pm$ ee $=2.5 \pm 1.7)$ y en machos entre uno y nueve $(\overline{\mathrm{X}} \pm$ ee $=2.8 \pm 1.7)$. No se encontró una relación entre la talla de las ranas y el número de presas consumidas $\left(\chi^{2}=\right.$ $8.05 \mathrm{E}^{-5}$; g.l. $\left.=64 ; \mathrm{P}=0.98\right)$, ni diferencias entre machos y hembras $\left(\chi^{2}=0.21 ; \mathrm{gl}=63 ; \mathrm{P}=0.46\right)$, pero sí se encontró un efecto de la interacción del sexo y la talla $\left(\chi^{2}=4 ;\right.$ g.l. $\left.=62 ; \mathrm{P}=0.01\right)$. En hembras se observó una asociación negativa 

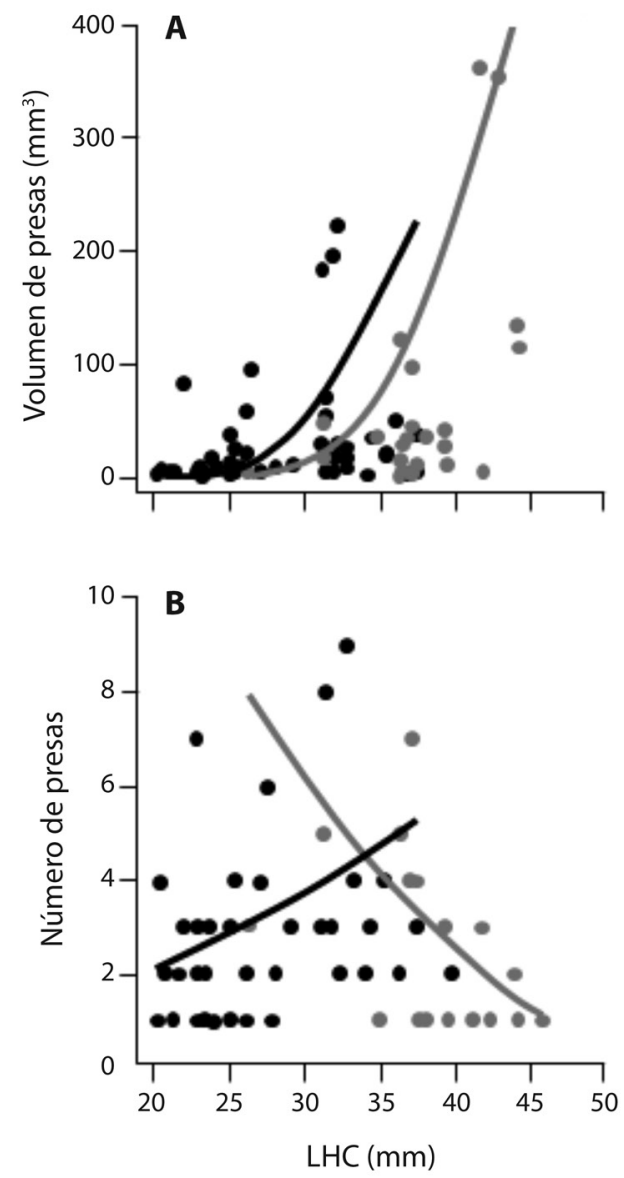

Fig. 3. Relación entre la LHC de individuos adultos de Craugastor rhodopis y A) volumen de cada presa consumida, y B) número de presas consumidas. Puntos y líneas de tendencia, gris representa a las hembras y negro a los machos.

Fig. 3. Relationship between the SVL of adult individuals of Craugastor rhodopis and A) volume of each prey consumed, and B) number of prey consumed. Gray points and trend lines represent females and black ones represent male.

entre estas variables (coef \pm ee $=-0.07 \pm 0.02$ ), siendo las hembras grandes las que consumen menor número de presas, mientras que en los machos la respuesta fue inversa (coef \pm ee $=$ $0.1 \pm 0.02$ ) siendo los machos grandes los que consumen más presas que los machos pequeños (Fig. 3B).

Finalmente, el número de categorías de presas consumidas por $C$. rhodopis osciló entre uno y siete $(\overline{\mathrm{X}} \pm$ ee $=2.3 \pm 1.3)$, los machos tuvieron entre uno y siete categorías $(\overline{\mathrm{X}} \pm$ ee $=2.5 \pm 1.4)$ y las hembras entre uno y cinco categorías $(\overline{\mathrm{X}} \pm \mathrm{ee}=2 \pm 1.2)$. No se encontró un efecto de la talla de los individuos sobre el número de categorías de presas $\left(\chi^{2}=0.9 ;\right.$ g.l. $=$ $64 ; \mathrm{P}=0.6)$, tampoco diferencias entre sexos $\left(\chi^{2}=0.48 ;\right.$ g.l. $\left.=63 ; \mathrm{P}=0.22\right)$, ni respuesta significativa de la interacción de la talla con el $\operatorname{sexo}\left(\chi^{2}=1.9 ;\right.$ g.1. $\left.=62 ; \mathrm{P}=0.1\right)$.

\section{DISCUSIÓN}

Los resultados de este estudio indican que la dieta de $C$. rhodopis en el centro de Veracruz está compuesta por un amplio número de categorías de presas, dentro de las cuales predominan los ortópteros. Esto sugiere que esta especie emplea principalmente una estrategia de forrajeo de acecho. Debido a su amplia composición dietaria, al bajo porcentaje de individuos sin alimento y su alta abundancia en los fragmentos del bosque de niebla, $C$. rhodopis podría estar desempeñando un papel importante en la dinámica de las comunidades de artrópodos. Debido a que la dieta de los machos estuvo compuesta por presas más pequeñas que en las hembras, los individuos de cada sexo podrían estar impactando de manera diferencial en las poblaciones de artrópodos.

Un amplio número de categorías de presas ha sido reportado en otras especies del mismo género (e.g. Craugastor lineatus en Chiapas, México; Martínez-Coronel \& Pérez-Gutiérrez, 2011) y de otros géneros de craugastóridos (e.g. Pristimantis labiosus; Gutiérrez-Cárdenas, Castillo, Martínez, Rocha, \& Rojas-Rivera, 2016). Adicionalmente, la predominancia de ortópteros en la dieta de C. rhodopis, parece ser un fenómeno común en los craugastóridos, pues en varios de ellos se encuentran entre las tres principales presas consumidas (Parmelee, 1999; Martínez-Coronel \& Pérez-Gutiérrez, 2011). Esto puede deberse a que los ortópteros son artrópodos móviles (Toft, 1980), presentan un cuerpo blando, con alto contenido energético y proteico (Anderson \& Smith, 1998). Además, son de mayor tamaño en comparación 
a otros artrópodos, por lo que tienen una menor proporción superficie-volumen, y por lo tanto, una menor cantidad relativa de quitina (Hirai, 2002).

Aunque la determinación del modo de forrajeo de $C$. rhodopis no estuvo dentro de los objetivos de este estudio, pues para esto sería necesario cuantificar la oferta de alimento, ciertas características morfológicas y de la dieta sugieren que esta especie emplea principalmente un modo de forrajeo de acecho, al igual que otras especies de craugastóridos (Toft, 1981; Parmelee, 1999). Dentro de sus características morfológicas se encuentran un cuerpo robusto con un hocico ancho y una coloración críptica, mientras que las relacionadas a la dieta incluyen un elevado número de categorías de presa y una predominancia de presas grandes de gran movilidad y de hábitos solitarios (e.g. ortópteros y coleópteros; Toft, 1981). Parmelee (1999) sugirió que un alto porcentaje de estómagos vacíos también está relacionado con una estrategia de acecho; sin embargo, C. rhodopis en el centro de Veracruz parece no ajustarse a esta predicción, pues presentó un bajo porcentaje de estómagos vacíos en comparación con otras especies de craugastóridos de bosques de niebla de México y selvas lluviosas premontanas de Colombia (Martínez-Coronel \& Pérez-Gutiérrez, 2011; Gutiérrez-Cárdenas et al., 2016). Alternativamente, es probable que esto se deba a que los machos no estaban involucrados en actividades reproductivas al momento de encontrarlos, o a una gran abundancia de presas en la época (nuestro estudio se llevó a cabo durante la época de lluvias) y sitios muestreados, tal como lo sugieren Biavati et al. (2004).

La composición de la dieta en $C$. rhodopis resultó ser similar entre sexos, al igual que en muchas otras especies de distintas familias de anuros (Biavati et al. 2004; Crnobrnja-Isailović et al., 2012; Rodrigues \& dos Santos-Costa, 2014). Sin embargo, se observaron algunas presas exclusivas en machos. Es probable que esto se deba a la marcada inequidad del tamaño de muestra entre sexos. A pesar de que una dieta similar puede sugerir la presencia de competencia intraespecífica por los recursos tróficos (Crnobrnja-Isailović et al., 2012), es probable que en $C$. rhodopis sea mínima, dadas las diferencias intersexuales en el tamaño de las presas; además del bajo número de individuos sin comida. El efecto de la talla de las ranas en el tamaño de las presas, en el que ranas más grandes consumieron presas de mayor tamaño, en comparación con las más pequeñas, podría ser resultado de una estrategia para cubrir la inversión energética designada a la reproducción, mayor en hembras y machos de mayor talla, como se ha reportado para otras especies (Chen, Tang, Fan, Wang, \& Pike, 2013; Finkler, Hayes, \& Rifai, 2014). El efecto significativo de la interacción del sexo y el tamaño de las ranas sobre el número de presas consumidas, sugiere que la competencia intraespecífica entre adultos podría ser mayor entre machos grandes y hembras pequeñas, ya que son de tamaño similar y consumieron mayor número de presas que las hembras grandes y los machos pequeños.

Este trabajo sienta las bases para estudios posteriores relacionados con otros aspectos de la ecología trófica de esta especie, como: 1) el efecto de la ontogenia, que es un factor determinante en la composición de la dieta de varios craugástoridos (Whitfield \& Donnelly, 2006), y 2) la composición de la dieta en función de la disponibilidad de las presas, con el fin de determinar la estrategia de forrajeo de la especie, y el grado de oportunismo y selección que tiene sobre los distintos tipos de presa. Asimismo, sería de gran relevancia 1) evaluar el grado de competencia intraespecífica que existe entre sexos y clases de edad, y 2) el efecto que tiene esta especie sobre las poblaciones de invertebrados que habitan la hojarasca, debido al número de categorías de presa que forman parte de su dieta.

Declaración de ética: los autores declaran que todos están de acuerdo con esta publicación y que han hecho aportes que justifican su autoría; que no hay conflicto de interés de cualquier tipo; y que han cumplido con todos los requisitos y procedimientos éticos y legales 
pertinentes. El documento firmado se encuentra en los archivos de la revista.

\section{AGRADECIMIENTOS}

Agradecemos a Policarpo Ronzón, Antonio Vázquez, Adriana Sandoval, Flor Vázquez, Yocoyani Meza, Amauri Sarmiento y Chanel Juárez por su ayuda en el trabajo de campo. A Paulina García por su ayuda en el análisis de los contenidos estomacales. A Sandra Rocha por facilitarnos equipo de laboratorio. Este estudio fue financiado con fondos del proyecto HK006-CONABIO.

\section{RESUMEN}

La composición de la dieta en anfibios puede ser influenciada por diversos factores que causan su variación intraespecífica, como pueden ser la distribución geográfica, ontogenia, estacionalidad, y el sexo de los organismos. El objetivo de este estudio fue analizar la composición de la dieta en adultos de la Craugastor rhodopis (rana hojarasquera común) en la región montañosa del centro de Veracruz, México. Un total de 77 individuos adultos fueron colectados durante la época de lluvias de 2012, de los cuales 66 tuvieron contenido estomacal. Se realizó un análisis de la variación intersexual en la diversidad de la dieta, y el volumen, número de presas, y número de categorías de presa consumidas. La dieta de C. rhodopis estuvo compuesta por 20 categorías, de las cuales predominó Orthoptera en cuanto a número, volumen, frecuencia de ocurrencia e importancia relativa. Los machos consumieron presas de menor tamaño en relación a las hembras. Aunque ambos sexos consumieron similar número de presas, se encontró un efecto significativo de la interacción del sexo y el tamaño de las ranas sobre esta variable de la dieta, en la cual las hembras pequeñas consumen más presas que las grandes, y los machos grandes consumen más presas que los pequeños. El alto consumo de ortópteros (al menos en cuanto a volumen) es un fenómeno común dentro de la familia Craugastoridae y posiblemente está relacionado con el éxito de algunas especies en los ecosistemas donde habitan. A pesar de que la composición de la dieta fue similar entre sexos en C. rhodopis, se sugiere que la competencia intersexual por los recursos tróficos es mínima, dadas las diferencias en el tamaño de presas consumidas por machos y hembras.

Palabras clave: anfibios; composición de la dieta; diversidad trófica; ecología trófica; modelos lineales; variación intersexual.

\section{REFERENCIAS}

Aguilar-López, J. L., \& Pineda, E. (2013). An exotic species of earthworm preyed by Craugastor rhodopis (Anura: Craugastoridae) in Mexico. Herpetology Notes, 6, 335-336.

Anderson, J. T., \& Smith, L. M. (1998). Protein and energy production in playas: implications for migratory bird management. Wetlands, 18, 437-446.

Arroyo, S. B., Serrano-Cardozo, V. H., \& Ramírez-Pinilla, M. P. (2008). Diet, microhabitat and time of activity in a Pristimantis (Anura, Strabomantidae) assemblage. Phyllomedusa, 7, 109-119.

Bates, D., Maechler, M., Bolker, B., \& Walker, S. (2015). Fitting linear mixed-effects models using lme4. Journal of Statistical Software, 67, 1-48.

Beard, K. H., Eschtruth, A. K., Vogt, K. A., Vogt, D. J., \& Scatena, F. N. (2003). The effects of the frog Eleutherodactylus coqui on invertebrates and ecosystem processes at two scales in the Luquillo experimental forest, Puerto Rico. Journal of Tropical Ecology, 19, 607-617.

Berazategui, M., Camargo, A., \& Maneyro, R. (2007). Environmental and seasonal variation in the diet of Elachistocleis bicolor (Guérin-Méneville 1838) (Anura: Microhylidae) from northern Uruguay. Zoological Science, 24, 225-231.

Biavati, G. M., Wiederhecker, H. C., \& Colli, G. R. (2004). Diet of Epipedobates flavopictus (Anura: Dendrobatidae) in a Neotropical Savanna. Journal of Herpetology, 38, 510-518.

Bonansea, M. I., \& Vaira, M. (2007). Geographic variation of the diet of Melanophryniscus rubriventris (Anura: Bufonidae) in northwestern Argentina. Journal of Herpetology, 41, 231-236.

Bull, E. L. (2003). Diet and prey availability of Columbia spotted frogs in Northeastern Oregon. Northwest Science, 4, 349-356.

Chen, W., Tang, Z. H., Fan, X. G., Wang, Y., \& Pike, D. A. (2013). Maternal investment increases with altitude in a frog on the Tibetan Plateu. Journal of Evolutionary Biology, 26, 2710-2715.

Clarke, K. R. (1993). Non-parametric multivariate analyses of changes in community structure. Australian Journal of Ecology, 18, 117-143.

Colwell, R. K. (2013). EstimateS: Statistical estimation of species richness and shared species from samples. Version 9. Retrieved from http://purl.oclc.org/ estimates

Colwell, R. K., Chao, A., Gotelli, N. J., Lin, S. Y., Mao, C. X., Chazdon, R. L., \& Longino, J. T. (2012). Models and estimators linking individual-based and 
sample-based rarefaction, extrapolation and comparison of assemblages. Journal of Plant Ecology, $5,3-21$.

Cope, E. D. (1867). Fifth contribution to the herpetology of tropical America. Proceedings of the Academy of Natural Sciences of Philadelphia, 18, 317-323.

Crawford, J. A., Shepard, D. B., \& Conner, C. A. (2009). Diet composition and overlap between recently metamorphosed Rana areolata and Rana sphenocephala: implications for a frog of conservation concern. Copeia, 2009, 642-646.

Crawley, M. J. (2007). The R book. London, UK: Imperial College London at Silwood Park.

Crnobrnja-Isailović, J., Ćurčić, S., Stojadinović, D., Tomašević-Kolarov, N., Aleksić, I., \& Tomanović, Z. (2012). Diet composition and food preferences in adult common toads (Bufo bufo) (Amphibia: Anura: Bufonidae). Journal of Herpetology, 46, 562-567.

Crump, M. L., \& Scott, N. J. (1994). Visual Encounter Surveys. En W. Heyer, M. A. Donnelly, R. A. McDiarmid, L. C. Hayec, \& M. C. Foster (Eds.), Measuring and Monitoring Biological Diversity: Standard Methods for Amphibians (pp. 84-92). Washington DC, USA: Smithsonian Institution Press.

Donnelly, M. A. (1991). Feeding patterns of the strawberry poison frog, Dendrobates pumilio (Anura: Dendrobatidae). Copeia, 1991, 723-730.

Finkler, M. S., Hayes, C. J., \& Rifai, L. (2014). Sexual dimorphisms in metabolism, organ mass, and reproductive energetics in pre-breeding American toads (Anaxyrus americanus). Copeia, 2014, 447-453.

Frost, D. R. (2018). Amphibian Species of the World: An Online Reference. Museo Americano de Historia Natural, Nueva York, EU. Retrieved from http:// research.amnh.org/herpetology/amphibia/index.html

Garcia, J. C., Posso-Gómez, C. E., \& Cárdenas-Henao, H. (2015). Diet of direct-developing frogs (Anura: Craugastoridae: Pristimantis) from the Andes of western Colombia. Acta Biológica Colombiana, 20, 79-87.

Gutiérrez-Cárdenas, P. D. A., Castillo, K., Martínez, D., Rocha, C. F. D., \& Rojas-Rivera, M. A. (2016). Trophic ecology of Pristimantis labiosus (Anura: Craugastoridae) from South-Western Colombia. North-Western Journal of Zoology, 12, 102-109.

Hirai, T. (2002). Ontogenetic change in the diet of the pond frog, Rana nigromaculata. Ecological Research, 17, 639-644.

Houston, D., \& Shine, R. (1993). Sexual dimorphism and niche divergence: feeding habits of the Arafura filesnake. Journal of Animal Ecology, 62, 737-748.
Lamb, T. (1984). The influence of sex and breeding condition on microhabitat selection and diet in the pig frog Rana grylio. The American Midland Naturalist, 111, 311-318

Lieberman, S. S. (1986). Ecology of the leaf litter herpetofauna of a neotropical rain forest: La Selva, Costa Rica. Acta Zoológica Mexicana, 15, 1-72.

Lima, A. P., \& Magnusson, W. E. (2000). Does foraging activity change with ontogeny? An assessment for six sympatric species of postmetamorphic litter anurans in Central Amazonia. Journal of Herpetology, 34, 192-200.

MacGregor-Fors, I., \& Payton, M. E. (2013). Contrasting diversity values: statistical inferences based on overlapping confidence intervals. PLOS ONE 8, e56794. DOI:10.1371/journal.pone.0056794

Magurran, A. E. (2004). Measuring Biological Diversity. Oxford, Reino Unido: Blackwell Publishing, Oxford.

Maneyro, R., Naya, D. E., da Rosa, I., Canavero, A., \& Camargo, A. (2004). Diet of the South American frog Leptodactylus ocellatus (Anura, Leptodactylidae) in Uruguay. Iheringia, Série. Zoologia, Porto Alegre, 94, 57-61.

Martínez-Coronel, M., \& Pérez-Gutiérrez, M. (2011). Composición de la dieta de Craugastor lineatus (Anura.Craugastoridae) de Chiapas, México. Acta Zoológica Mexicana, 27, 215-230.

Meza-Parral, Y., \& Pineda, E. (2015) Amphibian diversity and threatened species in a severely transformed neotropical region in Mexico. PLoSONE, 10(3), e0121652. DOI:10.1371/journal. pone.0121652

Miranda, T., Ebner, M., Solé, M., \& Kwet, A. (2006). Spatial seasonal and intrapopulational variation in the diet of Pseudis cardosoi (Anura: Hylidae) from the Araucária Plateau of Rio Grande do Sul, Brazil. South American Journal of Herpetology, 1, 121-130.

Murrieta-Galindo, R., López-Barrera, F., González-Romero, A., \& Parra-Olea, G. (2013). Matrix and habitat quality in a montane cloud-forest landscape: amphibians in coffee plantations in central Veracruz, Mexico. Wildlife Research, 40, 25-35.

Norval, G., Huang, S. C., Mao, J. J., Goldberg, S. R., \& Yang, Y. J. (2014). Notes on the diets of five amphibian species from southwestern Taiwan. ALYTES International Journal of Batrachology, 30, 69-77.

Parmelee, J. R. (1999). Trophic ecology of a tropical anuran assemblage. Scientific Papers of the Natural History Museum, the University of Kansas, 11, 1-59.

Pianka, E. R. (1973). The structure of lizard communities. Annual Review of Ecology and Systematics, 4, 53-74. 
Plummer, M. V., \& Farrar, D. B. (1981). Sexual dietary differences in a population of Trionyx muticus. Journal of Herpetology, 2, 175-179.

Quiroga, L. B., Sanabria, E. A., \& Acosta, J. C. (2009). Size- and sex-dependent variation in the diet of Rhinella arenarum (Anura: Bufonidae) in a wetland of San Juan, Argentina. Journal of Herpetology, 43, 311-317.

R Core Team. (2015). R: A language and environment for statistical computing. R Foundation for Statistical Computing, Vienna, Austria. Retrieved from http:// www.R-project.org

Rodrigues, L. C., \& dos Santos-Costa, M. C. (2014). Trophic ecology of Physalaemus ephippifer (Anura, Leptodactylidae) in Eastern Amazonia. Journal of Herpetology, 48, 532-536.

Rodríguez-Mendoza, C., \& Pineda, E. (2010). Importance of riparian remnants for frog species diversity in a highly fragmented rainforest. Biology Letters, 6, 781-784.

Streicher, J. W., García-Vázquez, U. O., Ponce-Campos, P., Flores-Villela, O., Campbell, J. A., \& Smith, E. (2014). Evolutionary relationships amongst polymorphic direct-developing frogs in the Craugastor rhodopis species group (Anura: Craugastoridae). Systematics and Biodiversity, 12, 1-22.

Toft, C. A. (1980). Feeding ecology of thirteen species of anurans in a seasonal tropical environment. Oecologia, 45, 131-141.

Toft, C. A. (1981). Feeding ecology of Panamanian litter anurans: patterns in diet and foraging mode. Journal of Herpetology, 15, 139-144.

Triplehorn, C. A., \& Johnson, N. F. (2005). Borror and DeLong's Introduction to the Study of Insects. Belment, USA: Thomson Brooks/Cole.
Urbina-Cardona, J. N., Olivares-Pérez, M., \& Reynoso, V. H. (2006). Herpetofauna diversity and microenvironment correlates across a pasture-edge-interior ecotone in tropical rainforest fragments in the Los Tuxtlas Biosphere Reserve of Veracruz, Mexico. Biological Conservation, 132, 61-75.

Urbina-Cardona, N., \& Reynoso, V. H. (2009). Uso del microhábitat por hembras grávidas de la rana de hojarasca Craugastor loki en la selva alta perennifolia de Los Tuxtlas, Veracruz. Revista Mexicana de Biodiversidad, 80, 571-573.

Warnes, G. R., Bolker, B., Lumley, T., \& Johnson, R. C. (2005). Contributions from Randall C. Johnson are Copyright SAIC-Frederick, Inc. Intramural Research Program, of the NIH, National Cancer Institute, Center for Cancer Research under NCI Contract NO1CO-12400. Retrieved from https://cran.r-project.org/ web/packages/gmodels/index.html

Whitfield, S. M., \& Donnelly, M. A. (2006). Ontogenetic and seasonal variation in the diets of a Costa Rican leaf-litter herpetofauna. Journal of Tropical Ecology, 22, 409-417.

Williams-Linera, G. (2007). El Bosque de niebla del centro de Veracruz: Ecología, historia y destino en tiempos de fragmentación y cambio climático. Xalapa, México: INECOL-CONABIO.

Williams-Linera, G., Manson, R. H., \& Isunza, E. V. (2002). La fragmentación del bosque mesófilo de montaña y patrones de uso del suelo en la región oeste de Xalapa, Veracruz, México. Madera y Bosques, 8, 73-89.

Zug, G. R., Vitt, L. J., \& Caldwell, J. P. (2001). Herpetology: An Introductory Biology of Amphibians and Reptiles. $2^{\text {nd }}$ Edition. San Diego, USA: Academic Press. 